\title{
Long-wave asymptotic theories: The connection between functionally graded waveguides and periodic media
}

\author{
R. V. Crastera , L. M. Joseph ${ }^{\mathrm{a}}$, J. Kaplunov ${ }^{\mathrm{b}}$ \\ ${ }^{a}$ Department of Mathematics, Imperial College London, London, SW7 2AZ, U.K. \\ ${ }^{b}$ School of Computing and Mathematics, Keele University, Keele, Staffordshire, ST5 5BG, UK
}

\begin{abstract}
This article explores the deep connections that exist between the mathematical representations of dynamic phenomena in functionally graded waveguides and those in periodic media. These connections are at their most obvious for low-frequency and long-wave asymptotics where well established theories hold. However, there is also a complementary limit of high-frequency long-wave asymptotics corresponding to various features that arise near cut-off frequencies in waveguides, including trapped modes. Simultaneously, periodic media exhibits standing wave frequencies, and the long-wave asymptotics near these frequencies characterise localised defect modes along with other high-frequency phenomena. The physics associated with waveguides and periodic media are, at first sight, apparently quite different, however the final equations that distill the essential physics are virtually identical. The connection is illustrated by the comparative study of a periodic string and a functionally graded acoustic waveguide.
\end{abstract}

Keywords: asymptotic, long-wave, low-frequency, high-frequency, homogenisation, waveguide, functionally graded, thin structure, trapped mode

\section{Introduction}

For long waves within a waveguide, at low-frequency, intuition suggests that (for waveguides governed by Helmholz equation with Neumann boundary conditions) the waveguide behaves effectively as a string; when viewed from afar the guide is long and thin. Similarly, a string composed of periodic elements where the length scales associated with the periodicity are much less than the wavelength of the excitation also intuitively behaves as some effective string. In both cases the word "effective" is rather vague, but as we shall see this can be made precise through an asymptotic approach involving twoscales; the thickness of the guide or periodicity scale and the length-scale of the guide or overall string. As we shall see an application of multiple scales leads rapidly to an effective equation for these two problems that can be simultaneously treated.

Perhaps less obviously one can also consider high-frequency wave propagation which almost immediately equates to short wavelength, as one typically thinks of waves within a bulk medium, and asymptotic techniques for waveguides based upon the WKBJ ansatz are popular and versatile $[1,2,3,4]$. However, the imposition of boundaries such as those of a waveguide can alter this intuitive viewpoint and long-wave solutions are also possible. Taking a straight, constant thickness, isotropic waveguide a natural approach Preprint submitted to Elsevier 
is to seek modal solutions and create dispersion curves. As is well-known (e.g. see [5]) there are an infinite set of discrete modes each with a cut-off frequency. For each mode the cut-off frequency delineates evanescent, exponentially decaying, solutions from the propagating modes. If one is exactly at the cut-off frequency (possibly at high-frequency) then this is called thickness resonance and the wave simply bounces back and forth across the waveguide and neither propagates to the left or right. The wavelength of this mode along the guide is actually infinite and close to cut-off the wavelength is therefore large. This observation motivates a general asymptotic methodology, summarized in the books $[3,6,7]$ in the context of thin elastic structures of arbitrary shape. In particular for the case of flat or axisymmetric waveguides with either weakly bent, bulging or thinning walls, $[8,9,10]$ ordinary differential equations (ODEs) for trapped modes (solutions with finite energy that decay exponentially at infinity) emerge. A key point is that in the deformed region one can shift the local cut-off frequency such that waves propagate locally, but are cut-off away from this region, thereby trapping modes. The simple ODE representation is then very powerful compared to large scale numerical eigenvalue calculations that lack insight.

A complementary, and apparently disconnected, area in wave propagation is that of waves passing through periodic media; this is important in solid-state physics [11], photonics [12] and the emergent areas of metamaterials [13]. For infinite perfectly periodic media, consisting of elementary cells that repeat, one can focus attention on a single elementary cell; quasi-periodic Floquet-Bloch boundary conditions describe the phaseshift as a wave moves through the material and dispersion relations are then deduced that relate the Bloch wavenumber, the phase-shift, to frequency. The eigensolutions that emerge are the Bloch modes, and when these eigensolutions are perfectly in-phase or out-of-phase across the cell then standing waves exist and the frequencies are then called standing wave frequencies (these frequencies can be high). There exist bands of frequencies, called band-gaps, in which propagating Bloch modes do not exist and in which the modes are evanescent. If the perfectly periodic lattice is perturbed then localised defect states can occur, these exponentially decay with distance, and the behaviour is eerily reminiscent of the trapped modes in a waveguide. Indeed at these high standing wave frequencies one can have "thickness resonance" within each elementary cell and the real wavenumber (not the Bloch wavenumber) is infinite as the wave itself is not propagating left or right. Asymptotic techniques based around high-frequency long-wave asymptotics have recently been developed [14] and ODEs in 1D periodic media (or PDEs in 2D) again emerge; this approach also works for microstructured discrete [15] or frame-like media [16]. The basic idea for periodic media is to replace the complicated microstructured medium with an equivalent, effective, continuum on a macroscale, that is, one wishes to homogenise the medium even when the wavelength and microstructure may be of similar scales.

We will illustrate the connection between the waveguide problem and periodic media by considering, in parallel, two model problems: A functionally graded acoustic waveguide and a periodic piecewise string. These are algebraically completely tractable and are explicitly solved in section 2 . The asymptotics of the low frequency model follow in section 3 and the linear asymptote emerges together with an effective string equation for both examples. The less intuitive case of high frequencies is dealt with in section 4 , there are only minor differences between the two examples but both feature a rapidly oscillating solution on one scale modulated by a long-scale function that satisfies an effective 
(a)

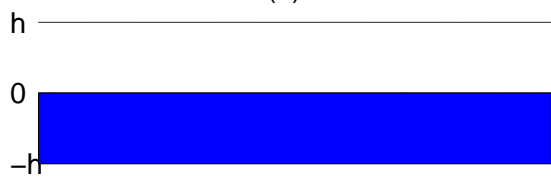

(b)

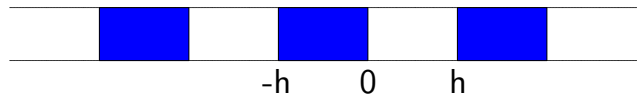

Figure 1: (a)Functionally graded waveguide and (b) Periodic string

equation posed entirely upon the long-scale. Explicit asymptotic results are found and compared with exact dispersion relations. The asymptotic techniques are also applied to deformed layered waveguides (section 5). Finally, we gather together some concluding remarks in section 6 .

\section{Formulation}

\subsection{Functionally graded waveguide}

Let us consider a straight waveguide, of constant width, in $|y|<h$ and $|x|<\infty$ with Neumann boundary conditions upon the waveguide walls, see Fig. 1(a). If the waveguide is elastic and excited by out-of-plane oscillations, so there is shear-horizontal (SH) polarisation, then the governing equation is that of acoustics with

$$
\left(\nabla^{2}+\frac{\omega^{2}}{\hat{c}^{2}(y / h)}\right) u(x, y)=0 .
$$

Notably we have allowed the wavespeed, $\hat{c}$, to vary across the waveguide and we take a reference wavespeed to be $c_{0}=\hat{c}(0)$ so $\hat{c}(y / h)=c_{0} c(y / h)$; the boundary condition is that $\partial u / \partial y=0$ on $y= \pm h$. In (1) the frequency is denoted by $\omega$.

We adopt the rescaling $\xi=y / h$ and $X=\epsilon x / h$ and the resultant non-dimensional governing equation is

$$
u_{\xi \xi}+\frac{\Omega^{2}}{c^{2}(\xi)} u+\epsilon^{2} u_{X X}=0
$$

with non-dimensional frequency $\Omega=\omega h / c_{0}$. The parameter $\epsilon$ is taken to be small, and can be identified with the ratio of the waveguide half-thickness $h$, to the longitudinal length-scale $L$, i.e. $\epsilon=h / L \ll 1$. The boundary condition on the waveguide walls is simply $u_{\xi}(X, \pm 1)=0$.

\subsection{Piecewise string}

Let us consider a string with wavespeed $\hat{c}(x / h)$, periodic with period $2 h$, and reference wavespeed $c_{0}=\hat{c}(0)\left(\hat{c}(x / h)=c_{0} c(x / h)\right)$ with governing equation

$$
u_{x x}+\frac{\omega^{2}}{\hat{c}^{2}(x / h)} u=0
$$

and now imagine that we have a long string composed of many of these periodic cells of width $2 h$, see Fig. 1(b). For clarity, we consider the simplest geometry for both problems where the wave speed varies linearly. It is, of course, possible to have different material variation leading to a more complicated functional form for the wave speed, in which 
case the treatment of the problem will be along the same lines: an example is presented by Craster et al. [14], who considered a string with periodic variation in density that had a trigonometric expression for the wave speed and in [20] where this is extended to two-dimensions.

We now adopt a multiple scales approach and assume that there is a short-scale $\xi=x / h$ and a long-scale $X=\epsilon x / h$ and hereafter $\xi, X$ are treated as independent coordinates. Physically, this assumes a strong separation of scales with a short, fine, microstructure characterised by the single periodic elementary cell of typical length $2 h$ and a longer macro-scale of typical length $L$ with $\epsilon=h / L \ll 1$ as in section 2.1.

For standing waves periodic across the structure, the non-dimensional equation in the new $(\xi, X)$ coordinates is then

$$
u_{\xi \xi}+\frac{\Omega^{2}}{c^{2}(\xi)} u+\epsilon^{2} u_{X X}+2 \epsilon u_{\xi X}=0
$$

with $u=u(X, \xi)$ and non-dimensional frequency $\Omega=\omega h / c_{0}$. Equation (4) is then identical to (2) except for the term of $2 \epsilon u_{\xi X}$.

The boundary conditions are that the solutions are in-phase (periodic) across the elementary cell, so $u(X, 1)=u(X,-1)$ and $u_{\xi}(X, 1)=u_{\xi}(X,-1)$. Notably this can be extended to deal with standing waves that are out-of-phase across the periodic cell in a straightforward manner [14]; for these we have $u(X, 1)=-u(X,-1)$ and $u_{\xi}(X, 1)=$ $-u_{\xi}(X,-1)$.

Except for the boundary conditions, and the single different term, the governing equations in sections 2.1 and 2.2 are identical and they can be dealt with together.

\subsection{Dispersion curves}

To fix ideas we consider a piecewise graded waveguide with $c(\xi)=1 / r$ for $0<\xi<1$ and $c(\xi)=1$ for $-1<\xi<0$. For simplicity the shear modulus of both media is taken to be identical. Assuming propagating modes such that $u(x, y)=\exp (i k x / h) \hat{u}(y)$ one rapidly arrives at a dispersion relation

$$
\begin{aligned}
& \left(\Omega^{2} r^{2}-k^{2}\right)^{\frac{1}{2}} \sin \left[\left(\Omega^{2} r^{2}-k^{2}\right)^{\frac{1}{2}}\right] \cos \left[\left(\Omega^{2}-k^{2}\right)^{\frac{1}{2}}\right]+ \\
& \left.\left(\Omega^{2}-k^{2}\right)^{\frac{1}{2}} \sin \left[\left(\Omega^{2}-k^{2}\right)^{\frac{1}{2}}\right] \cos \left(\Omega^{2} r^{2}-k^{2}\right)^{\frac{1}{2}}\right]=0,
\end{aligned}
$$

and the corresponding dispersion curves are shown in Fig. 2(a).

This is the analogous case to the piecewise periodic string treated in [14] for which the assumption is that Floquet-Bloch conditions, $u(1)=\exp (i 2 \kappa \epsilon) \hat{u}(-1)$ and $u_{\xi}(1)=$ $\exp (i 2 \kappa \epsilon) \hat{u}_{\xi}(-1)$ with $2 \epsilon$ being the relative width of the elementary cell, hold and the Kronig-Penney dispersion relation

$$
2 r[\cos \Omega \cos r \Omega-\cos 2 \epsilon \kappa]-\left(1+r^{2}\right) \sin \Omega \sin r \Omega=0
$$

follows relating the Bloch wavenumber, $\kappa$, to the frequency $\Omega$. It is clear that the wave numbers in (5) and (6) are linked as $k=\epsilon \kappa$. Dispersion curves are shown in Fig. 2(b). 
(a) Waveguide

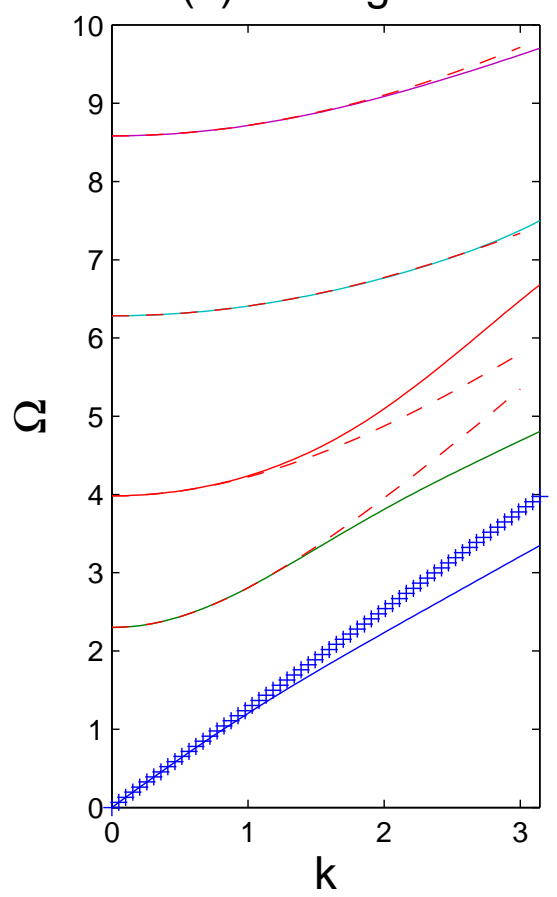

(b) Piecewise string

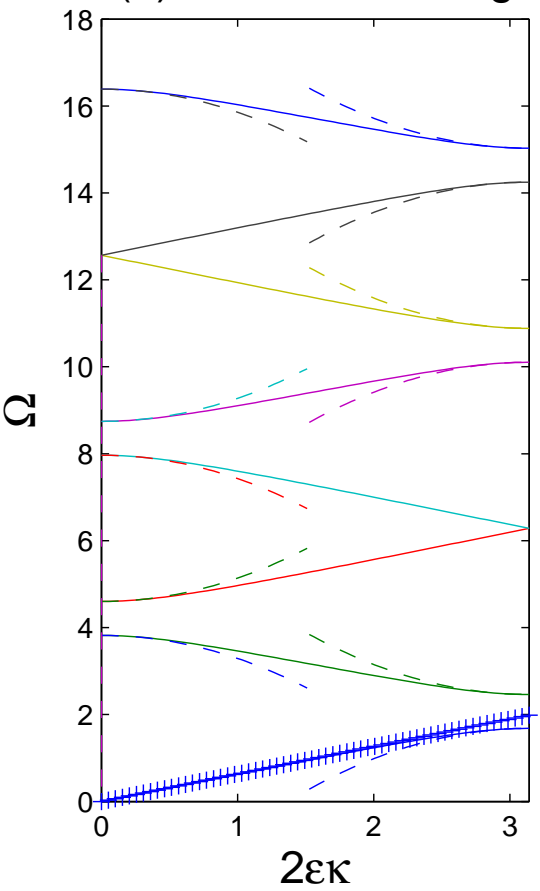

Figure 2: The dispersion curves for the bilayer waveguide and the piecewise string with $r=1 / 2$ : Numerics are the solid lines with the asymptotics as dashed and dotted lines, the linear classical asymptotics are the crosses. 


\section{The classical low-frequency limit}

At low frequencies, $\Omega^{2}=\epsilon^{2} \Omega_{2}^{2}+\ldots$, we have long waves, and the conventional lowfrequency viewpoint, and adopt the ansatz for $u$ that

$$
u(X, \xi)=u_{0}(X, \xi)+\epsilon u_{1}(X, \xi)+\epsilon^{2} u_{2}(X, \xi)+\ldots
$$

Inserting this ansatz into, say, (2) a hierachy of equations ensues:

$$
\begin{gathered}
u_{0 \xi \xi}=0, \quad u_{1 \xi \xi}=0, \\
u_{2 \xi \xi}=-\left(u_{0 X X}+\frac{\Omega_{2}^{2}}{c^{2}(\xi)} u_{0}\right)
\end{gathered}
$$

that are solved order-by-order together with the Neumann boundary conditions $u_{i \xi}(X, \pm 1)=0, i=0,1,2, \ldots$.

The leading order equation implies that $u_{0}(X, \xi)=u_{0}(X)$. This has the important corollary that the leading order field does not vary at all on the $\xi$ scale, i.e it is constant across the waveguide - or constant in each periodic cell in the periodic setting. Herein lies the inherent limitation of the traditional theories which cannot incorporate any local microscale, or cross guide, variation. Continuing up the orders, and invoking solvability, one eventually arrives at

$$
u_{0 X X}+\left\langle\frac{1}{c^{2}}\right\rangle \Omega_{2}^{2} u_{0}=0
$$

where

$$
\left\langle\frac{1}{c^{2}}\right\rangle=\frac{1}{2} \int_{-1}^{1} \frac{1}{c^{2}(\xi)} d \xi
$$

or in original variables

$$
h^{2} u_{0 x x}+\left\langle\frac{1}{c^{2}}\right\rangle \Omega^{2} u_{0}=0 .
$$

Assuming $u_{0}(x) \sim \exp (i k x / h)$, the lowest mode has a linear asymptote. In particular, for a piecewise graded waveguide it is

$$
\Omega \sim k \sqrt{\frac{2}{r^{2}+1}} .
$$

As one might anticipate, the governing equation is simply that of an effective onedimensional string with an averaged inverse wavespeed squared. This asymptotic is shown in Fig. 2(a,b) for both the waveguide and string.

\section{High-frequency limit}

A more interesting limit is the high-frequency, but long-wave, one. In this case $\Omega^{2}=\Omega_{0}^{2}+\epsilon \Omega_{1}^{2}+\epsilon^{2} \Omega_{2}^{2}+\ldots$. Again there are strong similarities in the asymptotic models. 


\subsection{Functionally graded waveguide}

As for the classical limit, we start from the anzatz (7). The hierarchy of equations now reads, for the waveguide, as

$$
\begin{gathered}
u_{0 \xi \xi}+\frac{\Omega_{0}^{2}}{c^{2}(\xi)} u_{0}=0, \\
u_{1 \xi \xi}+\frac{\Omega_{0}^{2}}{c^{2}(\xi)} u_{1}=-\frac{\Omega_{1}^{2}}{c^{2}(\xi)} u_{0}, \\
u_{2 \xi \xi}+\frac{\Omega_{0}^{2}}{c^{2}(\xi)} u_{2}=-\frac{\Omega_{1}^{2}}{c^{2}(\xi)} u_{1}-\frac{\Omega_{2}^{2}}{c^{2}(\xi)} u_{0}-u_{0 X X} .
\end{gathered}
$$

Here the Neumann boundary conditions at each order are the same as above. An immediate consequence of the leading order equation is that now

$$
u_{0}(X, \xi)=f_{0}(X) U_{0}\left(\xi ; \Omega_{0}\right)
$$

so, unlike the classical limit, there is now structure on the short-scale. The first order equation is trivial with $\Omega_{1}=0$ and $u_{1}$ can be set as an arbitrary multiple of $U_{0}$ and without loss of generality ignored. Notably, for the periodic string the first order problem is non-trivial, due to the $2 \epsilon u_{\xi X}$ term in (4), and an auxiliary equation, [14], needs to be introduced.

At second order, we get from solvability

$$
f_{0 X X} \int_{-1}^{1} U_{0}^{2} d \xi+\Omega_{2}^{2} f_{0} \int_{-1}^{1} \frac{U_{0}^{2}}{c^{2}} d \xi=0
$$

that is there is now an ODE for $f_{0}(X)$ entirely posed on the long-scale, $X$, and the short-scale features only through integrated quantities. In original variables

$$
h^{2} T f_{0 x x}+\left(\Omega^{2}-\Omega_{0}^{2}\right) f_{0}=0,
$$

where

$$
T=\frac{\int_{-1}^{1} U_{0}^{2} d \xi}{\int_{-1}^{1} U_{0}^{2} c^{-2} d \xi} .
$$

Performing the integrals, the asymptotic theory leads to the following asymptotic dispersion relation for a piecewise waveguide

$$
\Omega \sim \Omega_{0}+\frac{k^{2}}{2 \Omega_{0} r} \frac{r \delta(1)+\delta(r)}{\delta(1)+r \delta(r)},
$$

where

$$
\delta(s)=\frac{2 \Omega_{0} s+\sin \left(2 \Omega_{0} s\right)}{\cos ^{2}\left(2 \Omega_{0} s\right)},
$$

which is now quadratic and is compared to the full numerics in Fig. 2. 


\subsection{Piecewise string}

We obtain the following hierarchy of equations to be solved order-by-order

$$
\begin{gathered}
u_{0 \xi \xi}+\frac{\Omega_{0}^{2}}{c^{2}(\xi)} u_{0}=0, \\
u_{1 \xi \xi}+\frac{\Omega_{0}^{2}}{c^{2}(\xi)} u_{1}=-\frac{\Omega_{1}^{2}}{c^{2}(\xi)} u_{0}-2 u_{0 \xi X} \\
u_{2 \xi \xi}+\frac{\Omega_{0}^{2}}{c^{2}(\xi)} u_{2}=-\frac{\Omega_{1}^{2}}{c^{2}(\xi)} u_{1}-\frac{\Omega_{2}^{2}}{c^{2}(\xi)} u_{0}-u_{0 X X}-2 u_{1 \xi X}
\end{gathered}
$$

along with the boundary conditions $u_{i}(X, 1)= \pm u_{i}(X,-1)$ and $u_{i \xi}(X, 1)= \pm u_{i \xi}(X,-1)$, $i=0,1,2, \ldots$ with the plus and minus sign being for the periodic and anti-periodic conditions, respectively.

The solution to the leading order equation is the same as in the waveguide problem, see (17). In the first-order equation we observe the appearance of an extra term on the right hand side compared to the analogous equation in the waveguide case, which results in a solution different from that at first order. A solvability condition leads to $\Omega_{1}=0$, as before, and now

$$
u_{1}=f_{0 X}\left[A W_{1}\left(\xi ; \Omega_{0}\right)-\xi U_{0}\left(\xi ; \Omega_{0}\right)\right]+f_{1}(X) U_{0}\left(\xi ; \Omega_{0}\right)
$$

where $W_{1}\left(\xi ; \Omega_{0}\right)$ is determined to be a non-periodic solution of the leading order equation and

$$
A=\frac{2 U_{0}\left(1 ; \Omega_{0}\right)}{W_{1}\left(1 ; \Omega_{0}\right) \mp W_{1}\left(-1 ; \Omega_{0}\right)} .
$$

At next order, the solvability condition gives again the equation (19), where now

$$
T=\frac{2 A \int_{-1}^{1} U_{0} W_{1 \xi} d \xi}{\int_{-1}^{1} U_{0}^{2} c^{-2}(\xi) d \xi} .
$$

A degeneracy of this problem will occur whenever $\Omega_{0}$ is not a simple eigenvalue, since in this case the leading order solution (21) will consist of two linear independent periodic solutions: $u_{0}(X, \xi)=f_{0}^{(1)}(X) U_{0}^{(1)}\left(\xi ; \Omega_{0}\right)+f_{0}^{(2)}(X) U_{0}^{(2)}\left(\xi ; \Omega_{0}\right)$. By the solvability condition for the first-order term, we now obtain two coupled ODEs for $f_{0}^{(1,2)}$ from which the linear eigenvalue correction $\Omega_{1}^{2}$ is derived, [14],

$$
f_{0 X}^{(i)} \int_{-1}^{1} U_{0}^{(j)} U_{0 \xi}^{(i)} d \xi+\Omega_{1}^{2} \int_{-1}^{1}\left(f_{0}^{(j)} U_{0}^{(j) 2}+f_{0}^{(i)} U_{0}^{(i)} U_{0}^{(j)}\right) \frac{d \xi}{c^{2}(\xi)}=0
$$

for $i, j=1,2$ and $j \neq i$.

The effect of double eigenvalues was initially studied for thin elastic structures; see e.g. $[3,19]$.

The asymptotics developed are valid for $\Omega_{0}=\Omega_{\theta}$ near the edges of the Brillouin zone, where the Bloch wave number $\kappa=0(\theta=0)$ and $\kappa=\pi /(2 \epsilon)(\theta=\pi)$, and excluding 
the low-frequency fundamental mode passing through $\Omega_{0}=0$. We look back to the asymptotic procedure specifying it for a piecewise string, and in order to satisfy all the conditions we define $U_{0}\left(\xi ; \Omega_{\theta}\right)$ to be

$$
U_{0}\left(\xi ; \Omega_{\theta}\right)=\left\{\begin{array}{ccc}
\sin \left(r \Omega_{\theta} \xi\right)+p \cos \left(r \Omega_{\theta} \xi\right) & \text { for } \quad 0 \leq \xi<1 \\
r \sin \left(\Omega_{\theta} \xi\right)+p \cos \left(\Omega_{\theta} \xi\right) & \text { for } \quad-1 \leq \xi<0
\end{array}\right.
$$

for $p=\left(r \sin \Omega_{\theta} \pm \sin r \Omega_{\theta}\right) /\left(\cos \Omega_{\theta} \mp \cos r \Omega_{\theta}\right)$. The linearly independent solution, which does not satisfy periodicity-anti-periodicity conditions at $\xi= \pm 1$, is

$$
W_{1}\left(\xi ; \Omega_{\theta}\right)=\left\{\begin{array}{ccc}
\sin \left(r \Omega_{\theta} \xi\right) & \text { for } \quad 0 \leq \xi<1 \\
r \sin \left(\Omega_{\theta} \xi\right) & \text { for } \quad-1 \leq \xi<0
\end{array}\right.
$$

This function can be any solution of the leading order equation with fixed $\Omega_{\theta}$. By substituting these into (26), we deduce the $T=T_{0}$ and $T=T_{\pi}$ associated with periodic and anti-periodic solutions,

$$
T_{\theta}= \pm 4 \Omega_{\theta} \frac{\sin \Omega_{\theta} \sin r \Omega_{\theta}}{\left(r \sin \Omega_{\theta} \mp \sin r \Omega_{\theta}\right)\left(\cos \Omega_{\theta} \mp \cos r \Omega_{\theta}\right)}
$$

Considering the ODE for $f_{0}(x),(19)$, we set $f_{0} \sim \exp (i \epsilon \kappa x / h)$ for the periodic case and deduce that

$$
\Omega \sim \Omega_{0}+(\epsilon \kappa)^{2} \frac{T_{0}}{2 \Omega_{0}} .
$$

Similarly, in the anti-periodic case, we substitute $f_{0}(x) \sim \exp (i(\epsilon \kappa-\pi / 2) x / h)$

$$
\Omega \sim \Omega_{\pi}+(\epsilon \kappa-\pi / 2)^{2} \frac{T_{\pi}}{2 \Omega_{\pi}} .
$$

Numerical results based on the last two formulae are presented in Fig. 2.

We now turn our attention to the group velocity, $d \Omega / d \kappa$, at the cut-off frequencies in order to extract more information about the physics in those vicinities. The group velocity is defined for the periodic case as

$$
\frac{d \Omega}{d \kappa} \sim \epsilon^{2} \kappa \frac{T_{0}}{\Omega_{0}}
$$

and for the anti-periodic case as

$$
\frac{d \Omega}{d \kappa} \sim \epsilon(\epsilon \kappa-\pi / 2) \frac{T_{\pi}}{\Omega_{\pi}} .
$$

By looking at Table 1(a), we observe that the group velocity at the periodic end has the same sign as $T_{0}$, when this is positive the energy flow and the phase velocity are in the same direction ('regular' mode) conversely negative group velocity means the mode is 'backward', hence the wave appears to be travelling in the opposite direction to the energy. For the anti-periodic case, as shown in Table 1(b), the group velocity and the $T_{\pi}$ have alternating signs at the same frequency, but again the coefficient $T$ contains the essential information about the group velocity. The sign of the group velocity, along with other conditions, is intimately linked to the existence of trapped modes in elastic waveguides $[8,9]$. The coefficients $T_{0}, T_{\pi}$ therefore contain important information relevant to the energy flux via their relation to $d \Omega / d \kappa$. 
(a) Periodic case

\begin{tabular}{ccc}
\hline$\Omega$ & $T_{0}$ & $d \Omega / d \kappa$ \\
\hline 0 & + & + \\
3.8213 & - & - \\
4.6010 & + & + \\
7.9653 & - & - \\
8.7451 & + & + \\
16.3876 & - & - \\
\hline
\end{tabular}

(b) Anti-periodic case

\begin{tabular}{ccc}
\hline$\Omega$ & $T_{\pi}$ & $d \Omega / d \kappa$ \\
\hline 1.6821 & - & + \\
2.4619 & + & - \\
10.1045 & - & + \\
10.8842 & + & - \\
14.2485 & - & + \\
15.0283 & + & - \\
\hline
\end{tabular}

Table 1: Asymptotic results for the piecewise string shows the standing wave frequency, sign of $T$ and $d \Omega / d \kappa$ for non-repeated roots.

\section{High-frequency localisation}

As a further illustration, we briefly consider wave localisation for a waveguide that thickens or thins. For the sake of definiteness we assume the following variation of its thickness $H(\epsilon x / h)=h\left[1+0.5 \alpha \epsilon^{2} g(\epsilon x / h)\right]$, where $h$ and $\alpha$ are given constants, and the function $g$ may be taken, for example, as a Gaussian.

First, we introduce the variables $\xi=y /(H(\epsilon x / h))$ and $X=\epsilon x / h$. Then we get

$$
\left(\nabla^{2}+\frac{\Omega^{2}}{c^{2}(\xi)}\right) u(X, \xi)=0
$$

with

$$
\nabla^{2}=\frac{h^{2}}{H^{2}} \partial_{\xi \xi}+\epsilon^{2}\left\{\xi^{2}\left(\frac{H_{X}}{H}\right)^{2} \partial_{\xi \xi}-2 \xi \frac{H_{X}}{H} \partial_{\xi X}+\partial_{X X}+\xi\left[2\left(\frac{H_{X}}{H}\right)^{2}-\frac{H_{X X}}{H}\right] \partial_{\xi}\right\} .
$$

As in section 2.1, we adapt the ansatz (7) over the high-frequency domain, resulting in $(14,15)$ and also

$$
u_{2 \xi \xi}+\frac{\Omega_{0}^{2}}{c^{2}(\xi)} u_{2}=\alpha g u_{0 \xi \xi}-\frac{\Omega_{1}^{2}}{c^{2}(\xi)} u_{1}-\frac{\Omega_{2}^{2}}{c^{2}(\xi)} u_{0}-u_{0 X X}
$$

subject to Neumann boundary conditions at $\xi= \pm 1$ at each order.

Finally, we have

$$
f_{0 X X} \int_{-1}^{1} U_{0}^{2} d \xi+\left(\Omega_{2}^{2}+\alpha g \Omega_{0}^{2}\right) f_{0} \int_{-1}^{1} \frac{U_{0}^{2}}{c^{2}(\xi)} d \xi=0
$$

oriented to analysis of trapped modes similar to the defect states in a periodic chain [15], see also $[8,9,10]$. Numerical results of thickness variation of waveguides are presented in $[21,22]$, suggesting that the sign of the group velocity plays a crucial role in the existence of trapped modes, as well as curvature variation.

\section{Concluding remarks}

This comparative study illustrates a subtle analogue between the long-wave asymptotic procedures underlying approximate formulations for functionally graded wave guides and periodic media. This analogue is not restricted to the simplest setup assumed in the paper. The classical theories for thin plates, shells and rods seem to be a counterpart 
of the conventional low-frequency homogenisation theory. Similarly, the more advanced high-frequency long-wave structure theories $[3,6,7]$ have virtually the same philosophy as the recent high-frequency homogenisation procedure [14].

We also remark that the low-frequency limit is only possible for Neumann type boundary conditions. Other boundary conditions, including Dirichlet ones, do not support this limit enabling only high-frequency behaviour (e.g. see [17, 18] ). The high-frequency limit is also more universal in the case of periodic media (see the example in [14] for a periodic string resting on a stiff Winkler base).

\section{Acknowledgments}

RVC thanks the Engineering and Physical Sciences Research Council (EPSRC), UK, for their financial support through grant EP/J009636/1. LMJ thanks the A. G. Leventis Foundation.

\section{References}

[1] R. Burridge, H. Weinberg, Horizontal rays and vertical modes, in: J. B. Keller, J. S. Papadakis (Eds.), Wave propagation and underwater acoustics, Springer-Verlag, 1977, p. 86.

[2] R. Smith, Propagation in slowly varying wave-guides, SIAM J. Appl. Math. 33 (1972) 39-50.

[3] J. D. Kaplunov, L. Yu. Kossovich, E. V. Nolde, Dynamics of Thin Walled Elastic Bodies, Academic Press, New York, 1998.

[4] D. Gridin, R. V. Craster, Quasi-modes of a weakly curved waveguide, Proc. R. Soc. Lond. A 459 (2003) 2909-2931.

[5] J. G. Harris (Ed.), Elastic Waves at High Frequencies: Techniques for Radiation and Diffraction of Elastic and Surface Waves (Cambridge Monographs on Mechanics), Cambridge University Press, 2012, Ch. 8. Near cut-off behaviour in waveguides by R. V. Craster, pp. 133-146.

[6] V. L. Berdichevski, Variational principles of continuum mechanics, Nauka, Moscow, 1983, in Russian.

[7] K.C. Le, Vibrations of shells and rods, Springer, 1999.

[8] J. D. Kaplunov, G. A. Rogerson, P. E. Tovstik, Localized vibration in elastic structures with slowly varying thickness, Quart. J. Mech. Appl. Math. 58 (2005) 645-664.

[9] D. Gridin, R. V. Craster, A. T. I. Adamou, Trapped modes in curved elastic plates, Proc R Soc Lond A 461 (2005) 1181-1197.

[10] D. Gridin, R. V. Craster, A. T. I. Adamou, Trapped modes in bent elastic rods, Wave Motion 42 (2005) 352-366.

[11] C. Kittel, Introduction to solid state physics, 7th Edition, John Wiley \& Sons, New York, 1996.

[12] J. D. Joannopoulos, S. G. Johnson, J. N. Winn, R. D. Meade, Photonic Crystals, Molding the Flow of Light, 2nd Edition, Princeton University Press, Princeton, 2008.

[13] R. V. Craster, S. Guenneau (Eds.), Acoustic Metamaterials, Springer-Verlag, 2012.

[14] R. V. Craster, J. Kaplunov, A. V. Pichugin, High-frequency homogenization for periodic media, Proc R Soc Lond A 466 (2010) 2341-2362.

[15] R. V. Craster, J. Kaplunov, J. Postnova, High-frequency asymptotics, homogenization and localization for lattices, Q. Jl. Mech. Appl. Math. 63 (2010) 497-519.

[16] E. Nolde, R. V. Craster, J. Kaplunov, High-frequency homogenization for structural mechanics, J. Mech. Phys. Solids 59 (2011) 651-671.

[17] J.D.Kaplunov, Long-wave vibrations of a thin-walled body with fixed faces. Quart. J. Mech. Appl. Math. 48 (1995) 311-327.

[18] J.D.Kaplunov and E.V.Nolde, Long-wave vibrations of a nearly incompressible isotropic plate with fixed faces, Quart. J. Mech. Appl. Math. 55 (2002) 345-356.

[19] E.V. Nolde, Qualitative analysis of initial-value problems for a thin elastic strip, IMA J. Appl. Math., 72 (2007) 348-375.

[20] R. V. Craster, J. Kaplunov E. Nolde, S. Guenneau, High-frequency homogenization for checkerboard structures: defect modes, ultrafraction, and all-angle negative refraction, J. Opt. Soc. Am. A, 28 (2011) 1032-1040. 
[21] J. Postnova, R. V. Craster, Trapped modes in topographically varying elastic waveguides, Wave Motion 44 (2006) 205-221.

[22] J. Postnova, R. V. Craster, Trapped modes in elastic plates, ocean and quantum waveguides, Wave Motion 45 (2008) 565-579. 